\title{
Multisynchrony in active microfilaments
}

\author{
Yi Man and Eva Kanso* \\ Department of Aerospace and Mechanical Engineering, \\ University of Southern California, Los Angeles, California 90089, USA
}

(Dated: September 4, 2020)

\begin{abstract}
Biological microfilaments exhibit a variety of synchronization modes. Recent experiments observed that a pair of isolated eukaryotic flagella, coupled solely via the fluid medium, display synchrony at nontrivial phase-lags in addition to in-phase and anti-phase synchrony. Using an elasto-hydrodynamic filament model in conjunction with numerical simulations and a Floquet-type theoretical analysis, we demonstrate that it is possible to reach multiple synchronization states by varying the intrinsic activity of the filament and the strength of hydrodynamic coupling between the two filaments. We then derive an evolution equation for the phase difference between the two filaments at weak-coupling, and use a Kuramoto-style phase sensitivity analysis to reveal the nature of the bifurcations underlying the transitions between these different synchronized states.
\end{abstract}

Biological microfilaments, such as cilia and flagella, exhibit a variety of synchronization modes. As the surrounding fluid is an obvious medium for force transmission, hydrodynamic interactions are deemed crucial for synchronization. Two flagella isolated from the somatic cells of Volvox carteri, and later of Chlamydomonas reinhardtii, and thus coupled via the fluid medium only, synchronize their beating in-phase or anti-phase at close interflagellar distance [1, 2]. Synchronized states with nontrivial phase lags, between 0 and $\pi$, have also been observed, but not thoroughly analyzed [2].

Taylor pioneered the theoretical study of fluidmediated synchronization by considering two infinite sheets with prescribed traveling waves; he found that inphase synchronization is stable and exhibits minimum viscous energy dissipation [3]. Later, anti-phase synchrony, characterized by maximum dissipation, was also shown to be stable [4]. Synchronization was also analyzed in experiments with driven colloids [5-7] and in far-field models [8-11], assuming that the interfilamentous distance $h$ is much larger than the filament length $L$ so that each filament can be modeled as an oscillating bead. Due to time-reversibility of the Stokes equations, in addition to hydrodynamic coupling, either a non-constant force profile $[10,11]$ or orbital compliance [9] is necessary to achieve synchrony in the bead model. The synchronized state depends on the force profile and shape of the orbit. Particularly, for circular orbits, only in-phase synchrony is stable, while for select elliptic orbits, synchronized states with opposite phase or nontrivial phase lag appear to be stable $[8,10,11]$. Despite the richness of these weakly-coupled bead models, in most biological situations, the opposite regime where $h \ll L$ is more relevant and the slender geometry of the filament should be considered. Until recently, there are a few elastic filament models for synchronizations, and they mainly focus on in-phase and anti-phase synchronizations only [12-16].

Biological microfilaments, namely cilia and eukaryotic flagella, are driven into sustained oscillations by an in-

\footnotetext{
*kanso@usc.edu
}
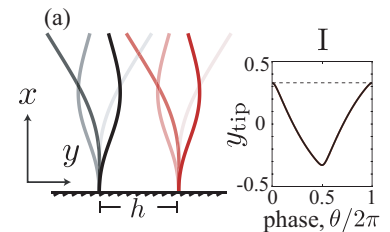

(c)

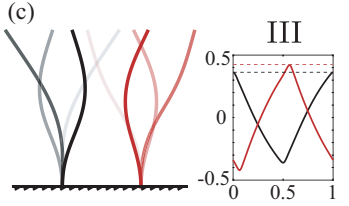

(b)

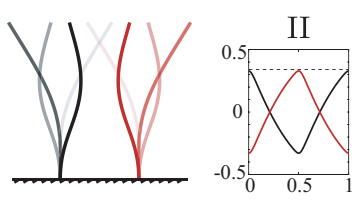

(d)

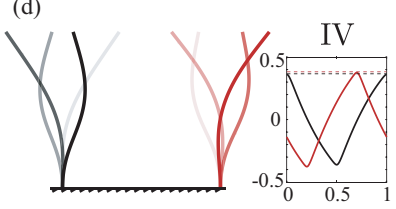

FIG. 1: Two active microfilaments synchronize their beating: (a) in-phase for $F=46, \gamma=0.1, \Delta \theta_{0} / 2 \pi=0.2$; (b) anti-phase for $F=46, \gamma=0.1, \Delta \theta_{0} / 2 \pi=0.3$; (c) at different amplitudes for $F=49, \gamma=0.1, \Delta \theta_{0} / 2 \pi=0.3 ;(\mathrm{d})$ at a nontrivial phase lag $(\Delta \theta \approx 0.56 \pi)$ for $F=48, \gamma=0.01, \Delta \theta_{0} / 2 \pi=0.3$. In each panel, left plots depict snapshots of the steady-state waveforms, with increasing time highlighted in darker color, and right plots show the tip deflection over one period of oscillation as a function of phase $\theta / 2 \pi$. These four cases, labeled I-IV, are highlighted in

Fig. $2 \& 3$.

tricate internal structure of microtubule doublets and dynein motors $[17,18]$. The spatial and temporal regulations of this molecular machinery are still under debate; see, e.g., [19-22] and references therein. We posit that the exact details driving flagellar oscillations matter little to the coordination of multiple flagella. In this Letter, we apply a recently-proposed phenomenological model, in which the active motor forces are represented by a tangential force $F$ exerted at the filament tip [2325]. We show that two filaments coupled via near-field hydrodynamics $(h \ll L)$ can reach multiple synchronized states with the same, opposite, and even nontrivial phase lags. We analyze the stability and basins of attraction of these states using Floquet theory and Kuramoto-style phase reduction analysis.

In particular, we consider two identical filaments of radius $a$ and length $L \gg a$, clamped at their base at a distance $h$ apart, and subject to an applied tangential force $F$ at their tip. We let $\mathbf{r}(s, t)$ denote the position 
of one of the filaments as a function of time $t$ and arclength $s$. The subscripts $(\cdot)_{t}$ and $(\cdot)_{s}$ denote differentiation with respect to $t$ and $s$, respectively. The hydrodynamic force density $\mathbf{f}_{\mathrm{h}}$ is anisotropic and proportional to the filament velocity relative to the fluid velocity; namely, $\mathbf{f}_{\mathrm{h}}=-\xi\left(\mathbf{I}-\frac{1}{2} \mathbf{t t}\right) \cdot\left(\mathbf{r}_{t}-\mathbf{v}\right)$. Here, we introduced the unit tangent $\mathbf{t}=\mathbf{r}_{s}$ andt used the approximation that the perpendicular drag coefficient $\xi=4 \pi \mu / \ln (L / a)$ is twice as large as the tangential drag coefficient $[26,27]$. The vector $\mathbf{v}$ represents the fluid velocity induced by the motion of the other filament; it is identically zero for a single filament. For planar motions (in the $(x, y)$ plane), it is a classic result that the elastic force is given by $\mathbf{f}_{\mathrm{e}}=-B \mathbf{r}_{\text {ssss }}+(\Lambda \mathbf{t})_{s}$, where $B$ denotes the bending rigidity and $\Lambda$ the tension enforcing filament inextensibility [28]. Balance of forces $\mathbf{f}_{\mathrm{h}}+\mathbf{f}_{\mathrm{e}}=\mathbf{0}$ on each filament, together with clamped-free boundary conditions, lead a system of equations for the filaments dynamics. We express this system in non-dimensional form by choosing the length scale $L$ and the time scale given by the bending relaxation time $\xi L^{4} / B$; the local force scales as $B / L^{3}$.

To close the system, we use $\mathbf{v}^{(j \rightarrow i)}$ to denote the flow field at filament $i$ induced by the motion of filament $j$. For $a \ll L$ and $a \ll h$, the fluid velocity can be represented by that induced by a line of Stokeslets distributed along the centerline [29]. In the biologically relevant limit $a \ll h \ll L$, Man and co-authors calculated that, at the leading order, $\mathbf{v}^{(j \rightarrow i)}(s)=(\ln (h(s) / L) / 4 \pi \mu)(\mathbf{I}+\mathbf{t t})$. $\mathbf{f}_{\mathrm{h}}^{(j)}(s)$, where $h(s)$ is the distance between two filaments at arc length $s$ [30-32]. We assume that the wave amplitude is at most of the same order as the basal interfilamentous distance $h$, and define $\gamma=\ln (L / h) / \ln (L / a)$ to indicate the strength of hydrodynamic coupling [12]. The flow velocity reduces to $\mathbf{v}^{(j \rightarrow i)}(s)=-\gamma \mathbf{r}_{t}^{(j)}(s)$. As the displacement in the $y$-direction is dominant, we relax the inextensibility condition and approximate $s \approx x$. The equations governing the coupled oscillations of the two filaments are given by

$$
\begin{aligned}
& y_{t}^{(1)}-\gamma y_{t}^{(2)}=-y_{x x x x}^{(1)}+2 \Lambda_{x}^{(1)} y_{x}^{(1)}+\Lambda^{(1)} y_{x x}^{(1)}, \\
& y_{t}^{(2)}-\gamma y_{t}^{(1)}=-y_{x x x x}^{(2)}+2 \Lambda_{x}^{(2)} y_{x}^{(2)}+\Lambda^{(2)} y_{x x}^{(2)},
\end{aligned}
$$

subject to the boundary conditions (for $i=1,2$ )

$$
\left.y^{(i)}\right|_{x=0}=\left.y_{x}^{(i)}\right|_{x=0}=\left.y_{x x}^{(i)}\right|_{x=1}=\left.y_{x x x}^{(i)}\right|_{x=1}=0 .
$$

We solve Eq. (1) numerically using an implicit finite difference scheme similar to that in [33]; see SI [34].

For a single filament, $\Lambda(x)=-F$ holds along the filament. As the active force $F$ exceeds a critical value $F_{\text {cr }}=37.5$, the filament buckles, and its linear dynamics is characterized by unstable oscillations with growing amplitude [23, 24]. To saturate the oscillation amplitude, we modify the tension by adding a nonlinear function of curvature, $\Lambda=-F+\alpha y_{x x}^{2}$, where the square comes from consideration of symmetry and $\alpha$ is a constant that we fix to $\alpha=4$. The beat frequency depends on $F$ not $\alpha$. The steady state behavior of the single filament follows a periodic, limit-cycle solution $y_{0}(x, t ; F)$, such that $y_{0}(x, t+T ; F)=y_{0}(x, T ; F)$; see SI and Fig. S1 [34]. We define a phase parameter $\theta$ such that the filament configuration can be parameterized by its phase in the oscillation cycle $y_{0}(s, \theta(t))$, and $\theta_{t}=\omega$, where $\omega / 2 \pi=1 / T$ is the oscillation frequency.

For two coupled filaments, we identify two periodic solutions $y^{(i)}(x, t ; F, \gamma)$ by direct inspection of Eq. (1): one solution $y^{(1)}=y^{(2)}=y_{0}(s, \theta), \theta_{t}=(1-\gamma)^{-1} \omega$ corresponds to the two filaments synchronizing in-phase and following the same waveform as that of a single filament albeit at a higher frequency; another solution, $y^{(1)}=-y^{(2)}=y_{0}(s, \theta), \theta_{t}=(1+\gamma)^{-1} \omega$ corresponds to anti-phase synchrony at a lower frequency. The fact that in-phase solutions exhibit higher beat frequencies is consistent with recent experimental observations and mathematical models $[2,13,35]$.

We initialize the two filaments at different phases $y^{(i)}(x, t=0 ; F, \gamma)=y_{0}\left(x, \theta_{0}^{(i)}\right)$ and solve Eq. (1) numerically. The steady state depends on the initial phase difference $\Delta \theta_{0}=\theta_{0}^{(2)}-\theta_{0}^{(1)}$ and the parameter values $F$ and $\gamma$. Interestingly, as we vary $\Delta \theta_{0}, F$ and $\gamma$, we find synchronization modes other than the in-phase and antiphase synchrony described above. In Fig. 1, we show four examples labeled I to IV. In I and II, the filaments converge to in- and anti-phase synchrony, respectively. In III, the filaments oscillate nearly out-of-phase at different amplitudes while in IV, the amplitudes are almost identical, and the filaments synchronize at a non-trivial phase lag, $\Delta \theta / 2 \pi=0.28$.

An analysis of the net hydrodynamic forces on the coupled filaments in Fig. 1 (see SI and Fig. S3 \& S4 [34]) shows that (i) compared to the single filament, the hydrodynamic force on each filament during in- and antiphase synchrony remains the same, (ii) asymmetric synchrony produces asymmetric forces that could result in a net moment on the filament pair, and (iii) the total force on both filaments is independent of the synchronization mode and coupling strength $\gamma$.

We next investigate the basins of attraction of these synchronization modes by systematically varying the initial phase difference $\Delta \theta_{0}$ for distinct values of $F$ and $\gamma$. In Fig. 2, we plot the results on the $\left(\Delta \theta_{0}, F\right)$ space, for $\gamma=0.01,0.1$ and 0.33 , which represent weak, intermediate, and strong hydrodynamic coupling, respectively. We observe that the dynamics strongly depends on $\gamma$. Under weak hydrodynamic coupling (Fig. 2a), all three synchronization modes are observed. For $F \lesssim 44$, the filaments are always synchronized anti-phase. For $45 \lesssim F \lesssim 48$, the filaments exhibit bistable behavior; they synchronize either in-phase or with nontrivial phase lags ranging approximately from $0.56 \pi$ to $\pi$, as represented by the color bar on the far right. For $F \gtrsim 49$, only in-phase synchrony is observed. Under intermediate coupling (Fig. 2b), the filaments exhibit bistable behavior for all $F$ with one transition: For $F \lesssim 48$, the filaments synchronize either in-phase or anti-phase, while for $F \gtrsim 49$, they synchro- 

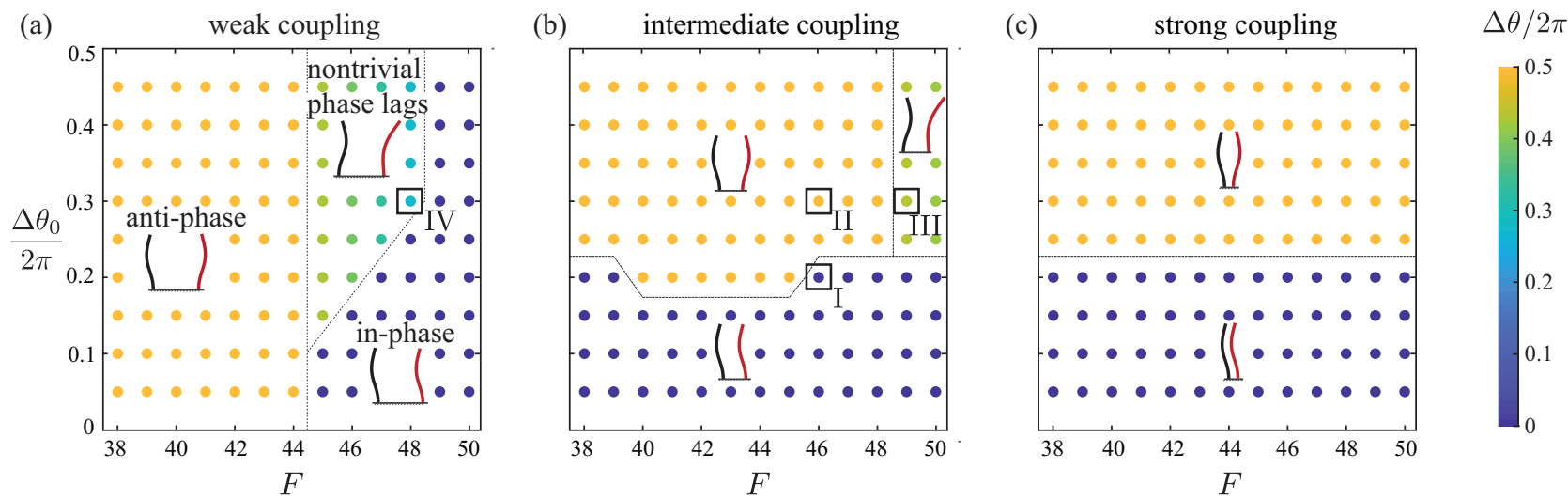

FIG. 2: Basins of attraction of the synchronization modes: anti-phase, in-phase, and synchrony at non-trivial phase lag, as a function of initial phase difference $\Delta \theta_{0}$ and the active force value $F$. The phase difference between two filaments at steady state, $\Delta \theta$, is indicated by the color of the dots. We vary the inter-filament spacing such that (a) $\gamma=0.01$, (b) $\gamma=0.1$ and (c) $\gamma=0.33$, representing weak, intermediate and strong hydrodynamic couplings.

nize either in-phase or at a nontrivial phase lag. For strong coupling (Fig. 2b), the filaments exhibit bistability between in-phase and anti-phase synchrony.

We analyze the stability of in-phase and anti-phase synchrony using Floquet theory. Considering first the case of in-phase synchrony $y^{(1)}=y^{(2)}=y_{0}(x, t ;(1-$ $\left.\gamma)^{-1} \omega\right)$, we add a perturbation $\delta y^{(i)}$ and substitute back into Eq. (1). The perturbations $\delta y^{(i)}$ are governed by the linear equations $\delta y_{t}^{(i)}-\gamma \delta y_{t}^{(j)}=\mathcal{L}\left[y^{(i)} ; y_{0}\right]$, where the right-hand side is given by $-y_{x x x x}+2[(-F+$ $\left.\left.\alpha y_{0 x x}^{2}\right) y_{x}+2 \alpha y_{0 x} y_{0 x x} y_{x x}\right]_{x}-\left(-F+3 \alpha y_{0 x x}^{2}\right) y_{x x}$. By linearity, the amplitude difference, $\delta y^{-}=\delta y^{(1)}-\delta y^{(2)}$, satisfies $(1+\gamma) \delta y_{t}^{-}=\mathcal{L}\left[\delta y^{-} ; y_{0}\right]$. Expressing in terms of the phase coordinate $\theta$, where $\theta_{t}=(1-\gamma)^{-1} \omega$, we arrive at the following linear equation about the in-phase state,

$$
\frac{1+\gamma}{1-\gamma} \omega \delta y_{\theta}^{-}=\mathcal{L}\left[\delta y^{-} ; y_{0}(x, \theta)\right]
$$

Similarly for the anti-phase state, we can derive an equation for the sum of perturbations, $\delta y^{+}=\delta y^{(1)}+\delta y^{(2)}$,

$$
\frac{1-\gamma}{1+\gamma} \omega \delta y_{\theta}^{+}=\mathcal{L}\left[\delta y^{+} ; y_{0}(x, \theta)\right]
$$

The solutions $\delta y^{ \pm}$are of the form $\delta y^{ \pm}=\delta y_{0}^{ \pm} e^{\mu t}$, where $\delta y_{0}^{ \pm}$is periodic and $\mu$ is the growth rate. We compute the associated Floquet multipliers $\rho \sim e^{\mu T}$ [36], by numerically integrating Eq. (2) and (3) over one period $T$. For $|\rho|<1,=1$ or $>1$, the corresponding synchronized state is stable, marginally stable, or unstable, respectively. In Fig. 3(b,c), we plot $|\rho|$ versus $\gamma$ for in-phase and antiphase synchrony, respectively, and for $F=42,46,48$ and 49. For $F=46, \gamma=0.1$, one has $|\rho|<1$ for both modes, consistently with cases I and II of Fig. 1. For $F=49$, $\gamma=0.1$ and for $F=48, \gamma=0.01$, in-phase synchrony is stable while anti-phase is not, as in cases III and IV. Further, the Floquet multipliers are consistent with all numerical predictions of in-phase and anti-phase stabil- ity reported in Fig. 2 (see SI and Fig. S3 [34]). Although this analysis does not shed light on the stability of the states with nontrivial phase lags, it does show that these states occur at values of $F$ and $\gamma$ for which anti-phase synchrony is unstable.

We posit that when synchrony is stable, the Floquet multiplier $\rho$ indicates the time it takes to synchronize. This time scales as $-1 / \mu \sim 1 / \ln |\rho|^{-1}(\mu<0$ for stable synchrony). In Fig. 3(a), we verify this finding numerically by calculating the number of periods until synchrony is reached in the nonlinear simulations and plotting versus $1 / \ln |\rho|^{-1}$. Small $|\rho|$ indicates fast synchronization. Interestingly, closer filaments with stronger hydrodynamic coupling (larger $\gamma$ ) do not always exhibit more efficient synchronization; while anti-phase synchronization is always achieved faster as the interfilamentous distance gets smaller (Fig. 3c), in-phase synchronization is most efficient at intermediate coupling (Fig. 3b).

To investigate the stability of all synchronized states,
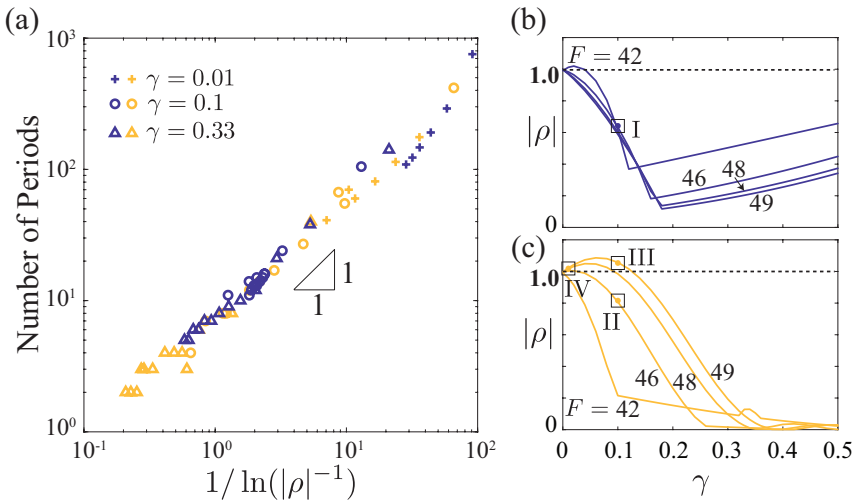

FIG. 3: (a) Number of periods it takes to reach in-phase (blue) or anti-phase (yellow) synchrony versus $1 / \ln \left(|\rho|^{-1}\right)$; symbols represent distinct values of $\gamma$ and $F$. Floquet multipliers versus $\gamma$ for $F=42,46,48$ and 49, corresponding to (b) in-phase and (c) anti-phase synchrony. 
(a)

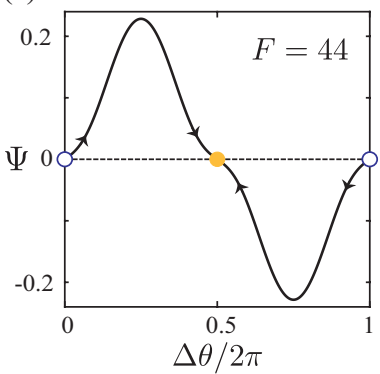

(b)

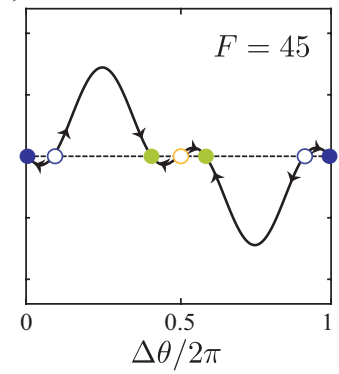

(c)

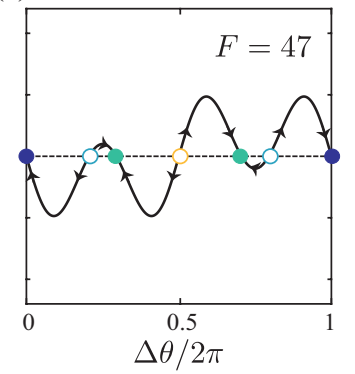

(d)

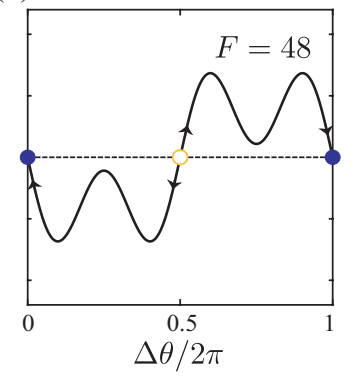

FIG. 4: Phase function $\Psi$ versus phase difference $\Delta \theta$ at (a) $F=44$, (b) 45 , (c) 47 and (d) 48 . The solid and hollow dots correspond to stable and unstable fixed points, respectively. The color scheme for $\Delta \theta$ uses the color map in FIG. 2 .

including those with nontrivial phase lag, we derive an evolution equation for the phase difference $\Delta \theta$ in the case of weak coupling. We use the Kuramoto phase reduction approach assuming that the dynamics of each filament asymptotically follows the single filament solution $y_{0}\left(x, t, \theta^{(i)}\right)$, albeit at a different phase [14].

We rewrite Eq. (1) in terms of the the eigenfunction $u_{0}\left(x, \theta^{(i)}\right)=\partial y_{0} /\left.\partial \theta\right|_{\theta=\theta^{(i)}}$ associated with the zero eigenvalue of the linear operator $(\mathcal{L}-\omega \partial / \partial \theta)$, and introduce the normalized adjoint function $\hat{u}_{0}(x, \theta)$ (see SI [34]). We project the resulting equation onto the single filament solution $u_{0}$ to obtain $\theta_{t}^{(i)}=\omega\left[1+\gamma H\left(\theta^{(i)}, \theta^{(j)}\right)\right]$, where $H\left(\theta^{(i)}, \theta^{(j)}\right)=\int_{0}^{1} \hat{u}_{0}\left(x, \theta^{(i)}\right) u_{0}\left(x, \theta^{(j)}\right) d x$ is the phase coupling function. We average $H$ over one cycle along the line $\theta^{(2)}=\theta^{(1)}+\Delta \theta$. The averaged $\bar{H}(\Delta \theta)$ depends only on the phase difference $\Delta \theta$. We arrive at the evolution equation

$$
\Delta \theta_{t}=\gamma \omega \Psi(\Delta \theta)
$$

where $\Psi(\Delta \theta)=\bar{H}(-\Delta \theta)-\bar{H}(\Delta \theta)$. Clearly, $\Psi(\Delta \theta)=0$ corresponds to equilibrium solutions of (4) for which the two filaments are in synchrony. The sign of $\partial \Psi / \partial(\Delta \theta)$ at these synchronized states indicates their stability: $\partial \Psi / \partial(\Delta \theta)$ is positive for unstable states and vice-versa.

In Fig. 4 , we plot $\Psi$ as a function of $\Delta \theta$ for $F=44$, 45,47 and 48 . These plots reveal two types of bifurcations underlying the transitions displayed in Fig. 2(a). At $F=44$, in-phase synchrony is unstable and antiphase synchrony is stable. Two supercritical pitchfork bifurcations take place as $F$ increases from 44 to 45 , by which the anti-phase synchrony becomes unstable and two stable equilibria appear at a nontrivial phase lag, and simultaneously, the in-phase synchrony becomes stable and two unstable equilibria appear at a nontrivial phase lag. The location of these equilibria changes with $F$. As $F$ increases from 47 to 48 , two saddle-node bifurcations occur and the nontrivial equilibria vanish leaving stable in-phase and unstable anti-phase synchrony.

Lastly, we examine key features observed in experiments with isolated flagella of Volvox carteri [1] in light of our filament model. Results taken from [1, Fig. 5A] are shown in Fig. 5(a); in-phase and anti-phase synchronous (a)
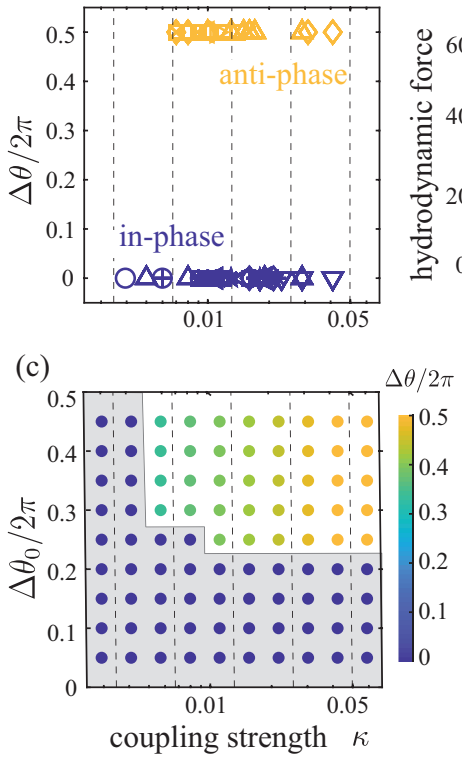

(b)

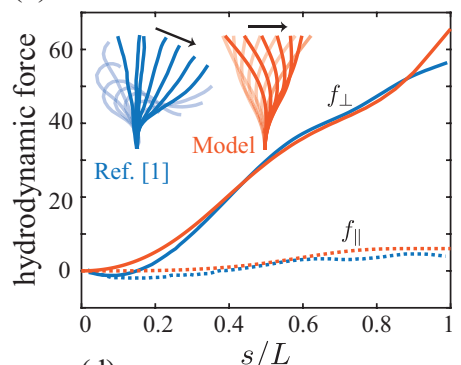

(d)

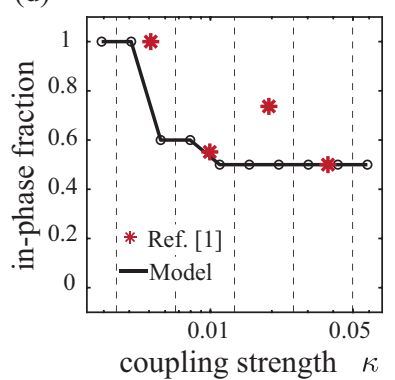

FIG. 5: (a) Experimental data from [1, Fig. 5A] represented in terms of steady-state phase difference $\Delta \theta / 2 \pi$ and coupling strength $\kappa$. (b) Normal (solid line) and tangential (dashed line) hydrodynamic forces based on the filament model with $F=50$ (orange) compared to flagellar forces (blue) [1, Fig. 4B]. Insets show snapshots of the filament waveform (orange) and flagellar waveform (blue) [1, Fig. 4A]. (c) Model prediction of synchronization modes for $F=50$ as a function of coupling strength $\kappa$. The grey region shows the basin of attraction of in-phase synchrony. (d) Fraction of in-phase basin of attraction to all initial phase differences; model results from (c) are shown in solid black line, experimental data from (a) are superimposed as red stars.

beating were reported for a range of coupling strength $\kappa$, where $\kappa$ is defined in terms of the time it takes for the two flagella to synchronize. Based on our Floquet analysis, $\kappa$ can be expressed as $\kappa=-\mu /(2 \pi \omega)$, where $-\mu=-\omega \ln \rho /(2 \pi)$ and $\rho$ depends on $F$ and $\gamma$. We arrive at $\kappa=\ln (1 / \rho) /\left(4 \pi^{2}\right)$, which for fixed $F$ defines a map from $\gamma$ to $\kappa$ (see SI [34]).

We first match the filament active force $F$ and frequency of oscillation $\omega / 2 \pi$ to those of the flagella. The measured flagellar frequency was about $30 \mathrm{~Hz}$ and total 
force about $50 \mathrm{pN}$ [1]. Using flagellar length $L=20 \mu \mathrm{m}$ and bending rigidity $B=4 \times 10^{-22} \mathrm{Nm}^{2}$, the dimensionless counterparts are $\omega / 2 \pi=48$ and $F=50$. For active force $F=50$ in our model, the resulting filament frequency $\omega / 2 \pi=46$ (Fig. S1,d) is close to that of the flagellar beat, and the distribution of forces along the filament and flagellum are also similar (Fig.5b).

In Fig. 5(c), we show the synchronization modes of a filament pair for the range of $\kappa$ reported in [1]. At small $\kappa$, only in-phase synchrony is stable. As $\kappa$ increases, both in- and anti-phase synchrony are stable, consistent with Fig. 2. The fraction of all initial phase differences (grey region in Fig. 5c) that lead to in-phase synchrony is shown in Fig. 5(d): fraction value 1 indicates that only inphase synchrony is stable while 0.5 means bistable in- and anti-phase synchrony with equal-size basins of attraction. To compare to Fig. 5(a), we interpret the experimental data as random samples from the phase space in Fig. 5(c), we divide $\kappa$ evenly in log-space into four ranges and we count the instances of in-phase synchrony in each range. The fraction of in-phase to total number of data points in each range are shown as red dots in Fig. 5(d). The results agree remarkably well with the filament model.

These findings could be instrumental for deciphering the biophysical and biochemical mechanisms underlying transitions in flagellar synchrony $[1,2,35]$. Such transitions could be triggered mechanically, say by random disturbances causing a shift between bistable modes, or physiologically by modifying either the intensity of the filament activity or interfilamentous coupling. The latter, in addition to hydrodynamics, could be due to basal connections between the flagella in the cell surface [3739]. These considerations, as well as extensions to arrays of microfilaments, will be treated in future works.
[1] Brumley DR, Wan KY, Polin M, Goldstein RE. Flagellar synchronization through direct hydrodynamic interactions. Elife. 2014;3:e02750.

[2] Wan KY, Kyriacos KC, Leptos C, Goldstein RE. Lag, lock, sync, slip: the many 'phases' of coupled flagella. J Royal Soc Interface. 2014;11:20131160.

[3] Taylor GI. Analysis of the swimming of microscopic organisms. Proc R Soc Lond A. 1951;209:447.

[4] Elfring GJ, Lauga E. Hydrodynamic phase locking of swimming microorganisms. Phys Rev Lett. 2009;103:088101.

[5] Kotar J, Leoni M, Bassetti B, Lagomarsino MC, Cicuta P. Hydrodynamic synchronization of colloidal oscillators. Proceedings of the National Academy of Sciences. 2010;107(17):7669-7673.

[6] Bruot N, Kotar J, de Lillo F, Lagomarsino MC, Cicuta P. Driving potential and noise level determine the synchronization state of hydrodynamically coupled oscillators. Physical Review Letters. 2012;109(16):164103.

[7] Bruot N, Cicuta P. Realizing the physics of motile cilia synchronization with driven colloids. Annual Review of Condensed Matter Physics. 2016;7(1):323-348.

[8] Vilfan A, Julicher F. Hydrodynamic flow patterns and synchronization of beating cilia. Phys Rev Lett. 2006;96:058102.

[9] Niedermayer T, Eckhardt B, Lenz P. Synchronization, phase locking, and metachronzal wave formation in ciliary chains. Chaos. 2008;18:037128.

[10] Uchida N, Golestanian R. Generic conditions for hydrodynamic synchronization. Phys Rev Lett. 2011;106:058104.

[11] Uchida N, Golestanian R. Hydrodynamic synchronzation between objects with cyclic rigid trajectories. EurPhysJ E. 2012;35:135.

[12] Goldstein RE, Lauga E, Pesci AI, Proctor MRE. Elastohydrodynamic synchronization of adjacent beating flagella. Phys Rev Fluids. 2016;1:073201.

[13] Guo H, Fauci L, Shelley M, Kanso E. Bistability in the synchronization of actuated microfilaments. J Fluid Mech. 2018;836:304.
[14] Kawamura T, Tsubaki R. Phase reduction approach to elastohydrodynamic synchronization of beating flagella. Phys Rev E. 2018;97:022212.

[15] Chakrabarti B, Saintillan D. Hydrodynamic synchronization of spontaneously beating filaments. Phys Rev Lett. 2019;123:208101.

[16] Stein DB, De Canio G, Lauga E, Shelley MJ, Goldstein RE. Swirling Instability of the Microtubule Cytoskeleton. arXiv e-prints. 2020 Aug;p. arXiv:2008.12018.

[17] Nicastro D, Schwartz C, Pierson J, Gaudette R, Porter ME, McIntosh JR. The molecular architecture of axonemes revealed by cryoelectron tomography. Science. 2006;313:944.

[18] Lin J, Nicastro D. Asymmetric distribution and spatial switching of dynein activity generates ciliary motility. Science. 2018;360:eaar1968.

[19] Bayly P, Dutcher S. Steady dynein forces induce flutter instability and propagating waves in mathematical models of flagella. Journal of The Royal Society Interface. 2016;13(123):20160523.

[20] Sartori P, Geyer VF, Howard J, Jülicher F. Curvature regulation of the ciliary beat through axonemal twist. Physical Review E. 2016;94(4):042426.

[21] Han J, Peskin CS. Spontaneous oscillation and fluidstructure interaction of cilia. Proc Natl Acad Sci. 2018;115:4417.

[22] Man Y, Ling F, Kanso E. Cilia oscillations. Phil Trans R Soc B. 2019;375:20190157.

[23] De Canio G, Lauga E, Goldstein RE. Spontaneous oscillations of elastic filaments induced by molecular motors. J Royal Soc Interface. 2017;14:20170491.

[24] Ling F, Guo H, Kanso E. Instability-driven oscillations of elastic microfilaments. J Royal Soc Interface. 2018;15:20180594.

[25] De Canio G. Motion of filaments induced by molecular motors: from individual to collective dynamics. University of Cambridge; 2018.

[26] Lauga E, Powers TR. The hydrodynamics of swimming microorganism. Rep Prog Phys. 2009;72:096601.

[27] Lighthill J. Flagellar hydrodynamics. SIAM review. 
1976;18(2):161-230

[28] Wiggins CH, Riveline D, Ott A, Goldstein RE. Trapping and wiggling: elastohydrodynamics of driven microfilaments. Biophysical Journal. 1998;74(2):1043-1060.

[29] Hancock GJ. The self-propulsion of microscopic organisms through liquids. Proc R Soc Lond A. 1953;217:96.

[30] Man Y, Lyndon K, Lauga E. Hydrodynamic interactions between nearby slender filaments. EPL. 2016;116:24002.

[31] Man Y, Page W, Poole RJ, Lauga E. Bundling of elastic filaments induced by hydrodynamic interactions. Phys Rev Fluids. 2017;2:123101.

[32] Man Y. Swimming at low Reynolds number: slip boundaries and interacting filaments. University of Cambridge; 2017.

[33] Tornberg AK, Shelley MJ. Simulating the dynamics and interactions of flexible fibers in Stokes flows. J Comput Phys. 2004;196:8-40.

[34] See Supplemental Material [url] for additional details of the model and numerical methods, force distributions, stability analysis, and comparison to experimental data, which includes Refs. [1, 12, 14, 23, 24, 27, 30, 31, 33, 40].

[35] Leptos KC, Wan KY, Polin M, Tuval I, Pesci AI, Gold- stein RE. Antiphase synchronization in a flagellardominance mutant of Chlamydomonas. Physical Review Letters. 2013;111(15):158101.

[36] Floquet G. Sur les équations différentielles linéaires à coefficients périodiques. Annales de l'École Normale Supérieure. 1883;12:27.

[37] Wan KY, Goldstein RE. Coordinated beating of algal flagella is mediated by basal coupling. Proceedings of the National Academy of Sciences. 2016;113(20):2784-2793.

[38] Liu Y, Claydon R, Polin M, Brumley DR. Transitions in synchronization states of model cilia through basalconnection coupling. Journal of The Royal Society Interface. 2018;15(147):20180450.

[39] Guo H, Man Y, Wan KY, Kanso E. Intracellular coupling modulates biflagellar synchrony. arXiv e-prints. 2020 Aug;p. arXiv:2008.07626.

[40] Polin M, Tuval I, Drescher K, Gollub JP, Goldstein RE. Chlamydomonas swims with two "gears" in a eukaryotic version of run-and-tumble locomotion. Science. 2009;325(5939):487-490. 